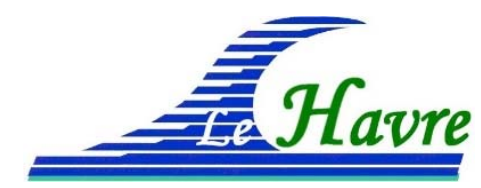

XVI èmes Journées Nationales Génie Côtier - Génie Civil

Le Havre, 2020

DOI:10.5150/jngcgc.2020.073 (C) Editions Paralia CFL

disponible en ligne - http://www.paralia.fr - available online

\title{
La gestion des sédiments et le développement de l'économie circulaire en Algérie
}

\author{
Fatiha KAZI AOUAL-BENSLAFA ${ }^{1}$, Kawther TOUHAMI ${ }^{1}$ \\ 1. Laboratoire LabMat, ENPOran, BP 1523, Oran El Ménaouer,31000 Oran, Algérie. \\ fkaziaoual@yahoo.fr ; fatiha.kazi-aoual@enp-oran.dz ; touhamikawther@gmail.com
}

\section{Résumé :}

Aujourd'hui de par le monde, la diminution des ressources naturelles et la préservation de l'environnement sont les préoccupations majeures des responsables. Le modèle économique basé sur la production de richesse par la destruction des ressources avec une surproduction de déchets a montré ses limites. Pour cela, de nouveaux principes apparaissent comme l'économie circulaire et au cœur de celle-ci, il y a le tri et le recyclage. Les gouvernements voient l'intérêt de l'économie circulaire et cela se traduit par des lois et des politiques qui ont vu le développement de stratégies de production d'éco-matériaux à partir des déchets industriels ou des déchets naturels tels que les sédiments de dragage. En Algérie, si la gestion des déchets a connu une évolution dans les domaines scientifique, technique et de mise en œuvre, le concept d'une économie circulaire reste à développer. Dans ce travail, un état des lieux est réalisé pour permettre un éclairage sur les enjeux et les perspectives de la gestion des déchets optimale.

\section{Mots-clés :}

Déchets-Sédiments, Valorisation, Environnement, Durabilité, Economie Circulaire.

\section{Introduction}

Aujourd'hui de par le monde, la diminution des ressources naturelles et la préservation de l'environnement sont les préoccupations majeures des responsables. Toutes les analyses des différentes recherches et publications scientifiques confirment que la diminution des ressources naturelles, la pollution de l'eau, de l'air et des sols et l'utilisation excessive des terres entrainent une dégradation de l'environnement. Le changement climatique, la perte de la biodiversité ou les inégalités sociales sont les impacts notables auxquels sont confrontés les états (LENZENet al., 2012; WWF, 2014). Alors, des changements sont nécessaires pour assurer une transition possible de la durabilité (La Conférence internationale sur le climat à Paris (COP21, 2015). L'économie circulaire (EC) émerge comme la nouvelle approche de la durabilité, elle est identifiée comme une alternative prometteuse à la logique linéaire (extraire - transformer - consommer - jeter), (MURRAYet al., 2017). La gestion durable des matériaux est une stratégie pour dissocier la croissance économique de la consommation des ressources naturelles et est définie par l'Organisation de coopération et de développement économiques (OCDE) comme "une approche pour promouvoir l'utilisation durable des matériaux, intégrant des actions visant 


\section{Thème 6 - Gestion durable des zones littorales et estuariennes}

à réduire les impacts environnementaux négatifs et à préserver le capital naturel tout au long du cycle de vie des matériaux, en tenant compte de l'efficacité économique et de l'équité sociale". Les gouvernements voient également l'intérêt de l'économie circulaire et cela se traduit par des lois et des politiques, auxquelles s'ajoutent de nombreux programmes, des objectifs, des feuilles de route et d'autres actions nationales et régionales : par exemple, Le Japon met en place la Loi pour la promotion d'une utilisation plus efficace des ressources (2000), la Chine avec sa Loi sur l'EC (2009), l'Allemagne vise à garantir une gestion écologiquement rationnelle des déchets (2012), le Green Deal du gouvernement néerlandais (2013), la Loi relative à la transition énergétique pour la croissance verte en France (2015) et, enfin, le train de mesures de la Commission européenne sur l'économie circulaire (2015), (NAZET-ALLOUCHE, 2016). Les organisations non universitaires qui ont en premier pris en charge l'économie circulaire, par exemple, la Fondation Ellen Mac Arthur au Royaume-Uni (2010), 1'Institut de l'économie circulaire en France (2013) et Circle Economy au Pays-Bas (2014). Les recherches se sont développées autour du concept de l'EC (MERLIet al., 2018 ; KAZI AOUAL - BENSLAFA \& TOUHAMI, 2019). Dans ce travail, nous présentons le concept de l'EC, ensuite une revue des principales recherches menées en Algérie sur la préservation de la ressource en eau et la gestion des sédiments de dragage et enfin nous présentons quelques perspectives pour aller vers une gestion optimale et durable.

\section{Le concept d'Economie Circulaire}

L'idée de l'économie circulaire (EC) n'est pas nouvelle. Le concept d'EC, apparu dans les années 70, ne s'est développé qu'à partir de 2010. Il prône un système économique en forme de boucle, il est présenté comme une voie de transition économique possible (KORHONENet al., 2018).Le cycle des matériaux est une caractéristique déterminante des systèmes biologiques, l'évolution continue des systèmes est garantie lorsque les anciennes structures sont décomposées et assimilées en de nouvelles. Le principe applique des analogies éco-systémiques à la conception des produits et de l'environnement bâti. Un système de production qui vise à recycler les matériaux lorsqu'ils atteignent leur fin de vie perçue. Alors, les déchets sont toujours la matière première d'un nouveau cycle et, en tant que tels, les ressources ne sont jamais gaspillées (BRENNAN et al., 2015). L'objectif principal de cette approche est de "découpler la croissance économique de l'épuisement des ressources naturelles et de la dégradation de l'environnement" (PAULIUK, 2018). Plusieurs écoles de pensée ont développé le concept de 1'EC (EMF-Ellen MacArthur Fondation, 2013) parmi elles, les approches du "Cradle to Cradle" $(\mathrm{C} 2 \mathrm{C})$ où les déchets deviennent une ressource génératrice de valeur $(\mathrm{Mc}$ DONOUGH et al., 2003). Le concept $\mathrm{C} 2 \mathrm{C}$ comprend deux sphères représentées en figure 1 , la biosphère comprenant des matériaux biodégradables et la techno-sphère comprenant des matériaux fabriqués par l'homme. 


\section{XVİ̀mes Journées Nationales Génie Côtier - Génie Civil \\ Le Havre 2020}

Pour l'Agence française de l'environnement et de la maîtrise de l'énergie (ADEME, 2014) l'économie circulaire repose sur trois piliers: la gestion des déchets, l'offre d'acteurs économiques et le comportement des utilisateurs. Pour obtenir des résultats significatifs, ce modèle économique ne doit négliger aucun de ces trois piliers (figure 2).

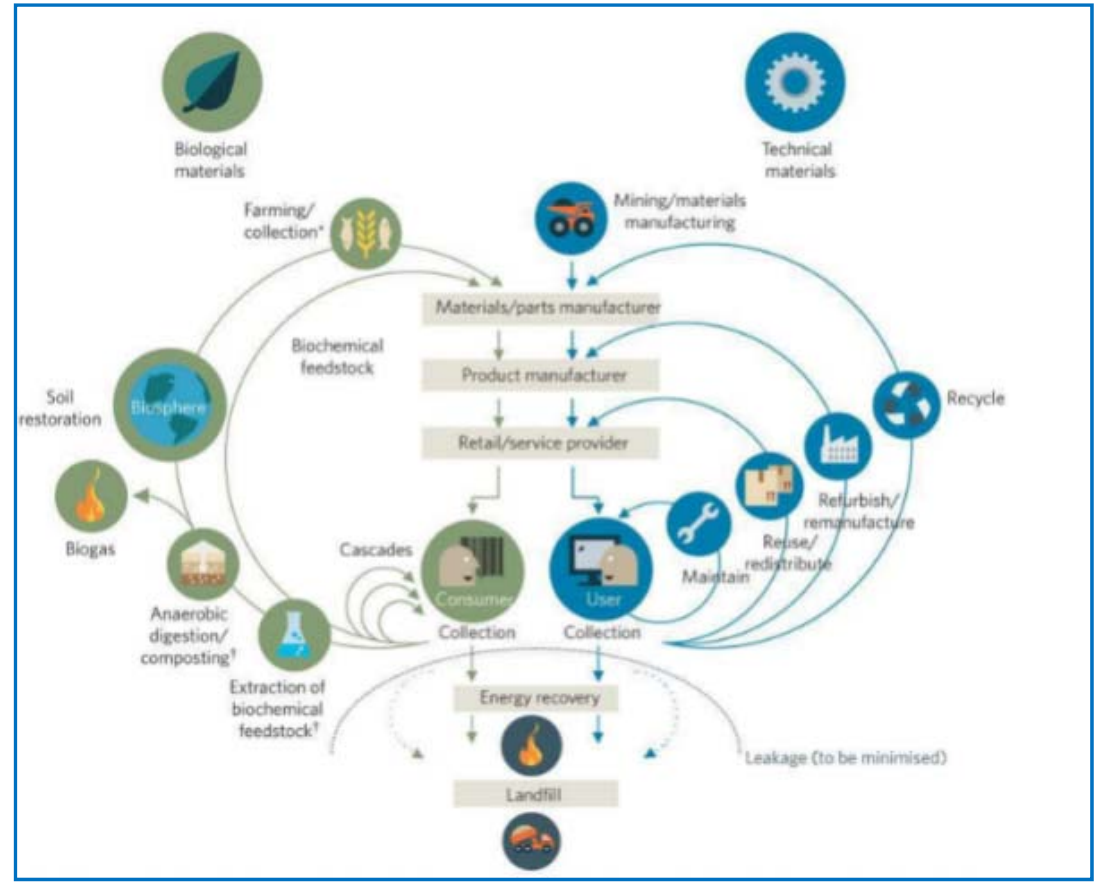

Figure 1. L'économie circulaire, (McDONOUGH et al., 2003).

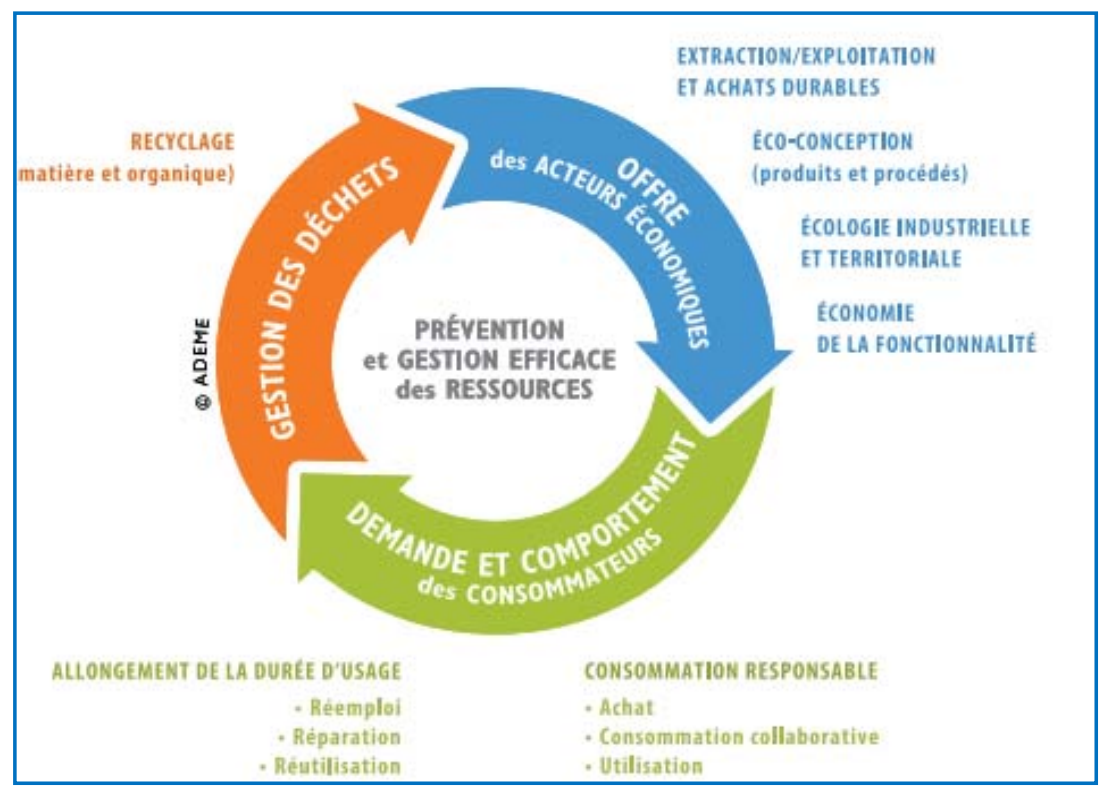

Figure 2. Composantes de l'économie circulaire: 3 piliers et 7 domaines d'action, (ADEME,2014). 


\section{Thème 6 - Gestion durable des zones littorales et estuariennes}

\section{La gestion des sédiments de dragage des barrages}

L'Algérie comme beaucoup de pays des régions semi arides, doit faire face au problème de la diminution des volumes d'eau dans les retenues de barrages causée par leur envasement. Celui-ci a atteint 1.7 milliards de $\mathrm{m}^{3}$ dans les 74 barrages en exploitation d'après les données de l'Agence Nationale des Barrages et Transfert (Agence Nationale des Barrages et Transferts - ANBT, 2015).

Le changement climatique dans ces régions va aggraver le phénomène d'envasement. Car l'irrégularité spatiale et temporelle très importante des pluies constitue ainsi une menace constante pour le remplissage des barrages réservoirs (TOUAHIR et al., 2018).

En plus, le réseau hydrographique est devenu saturé aujourd'hui et plus particulièrement dans l'ouest Algérien. Les sites favorables à la réalisation de nouveaux barrages se raréfient (REMINI, 2019). L'entretien des fonds des retenues des barrages par le dragage est une solution pour lutter contre l'envasement. Mais ces opérations de curage génèrent de grandes quantités de sédiments qui représentent un problème environnemental majeur. L'exemple du dragage du barrage de Fergoug illustre le dommage environnemental qui a été causé sur la plaine de la Macta. Dans le projet national auquel notre équipe a participé, nous nous sommes intéressés à l'étude du bassin versant de la Macta avec les trois barrages en cascade, le barrage d'Ouizert est faiblement envasé (10\%), les barrages de Fergoug et de Bouhanifia sont fortement envasés avec un taux de $94 \%$ et $56 \%$ respectivement. Nous évoquons dans ce qui suit les situations des deux barrages Fergoug et Bouhanifia.

Le barrage de Fergoug : les opérations de maintenance et d'entretien des fonds ont été réalisées en 1992 et en 2005 sur ce barrage et ont eu un impact négatif sur l'environnement et la dégradation des terres agricoles sur les zones de rejet. Les sédiments dragués ont été rejetés en aval de la digue provoquant la dégradation de l'environnement sur toute la plaine de la Macta.

Tous les résultats des recherches, ont montré que les sédiments dragués sont des sols fins composés d'argile et de sable fin en faible quantité, l'argile $(\approx 15 \%)$ ainsi que des sables fins $(<15 \%)$ et de limon en forte proportion $(>70 \%)$. Les sédiments dragués lorsqu'ils sont déposés sur les terres agricoles, forment une couche appelée "croûte de battance" qui empêche la croissance des plantes (LE BISSONNAIS et al., 1995).

La solution qui a été adoptée est celle d'une économie linéaire qui ne prenait pas en compte l'impact environnemental. Le modèle appliqué est "cradle to grave" (du "berceau à la tombe"). La figure 3 illustre le processus de la gestion des sédiments du barrage du Fergoug (SEMCHA et al., 2015) que l'on peut représenter sous forme du schéma représenté en figure 4, l'érosion du bassin versant par transport solide les sédiments sont déposés dans le lit de l'oued et dans la retenue du barrage. La deuxième étape est le dragage, les sédiments sont dragués mais ils sont considérés comme déchets ultimes, ils ne subissent aucun traitement et sont déposés en aval du barrage dans l'oued et par transport solide et entrainement se sont déposés sur la plaine de la Macta. 


\section{XVİ̀mes Journées Nationales Génie Côtier - Génie Civil \\ Le Havre 2020}
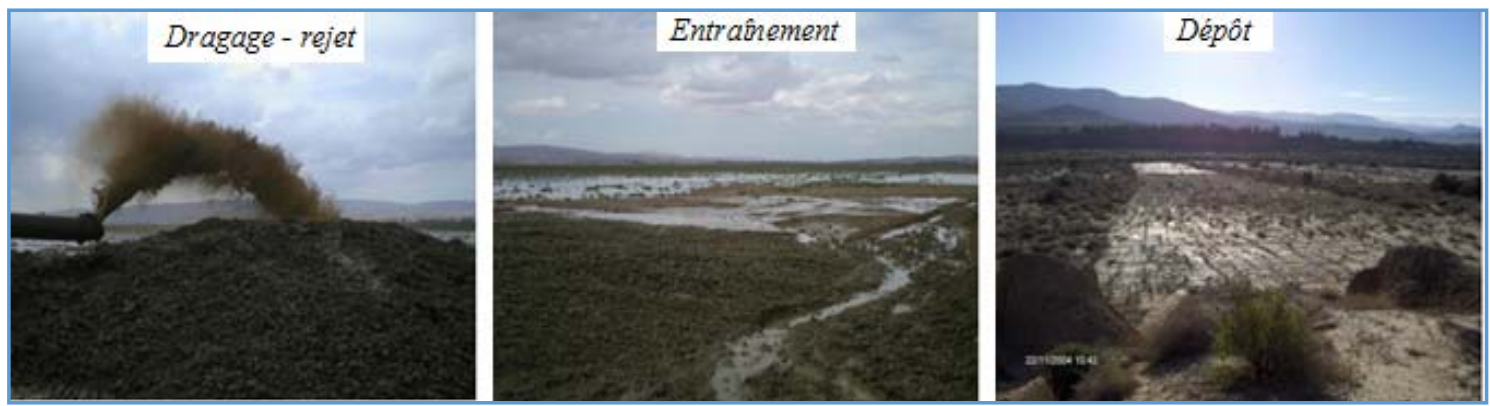

Figure 3. Erreurs de prise en charge des vases draguées à Fergoug,

(SEMCHA et al., 2015).

\begin{tabular}{|c|c|c|c|c|c|c|}
\hline \multirow{2}{*}{$\begin{array}{l}\text { Bassin } \\
\text { Versant }\end{array}$} & Transport & \multirow{2}{*}{ Barrage } & Dragage & \multirow{2}{*}{$\begin{array}{c}\text { Décharge sauvage } \\
\text { L'Oued }\end{array}$} & Transport & Déchet Ultime \\
\hline & solide & & et Rejet & & solide & Dépôt en Plaine \\
\hline
\end{tabular}

Figure 4. Gestion des sédiments de dragage du barrage du Fergoug.

Le barrage de Bouhanifia fait l'objet d'opérations de dragage depuis 2013, pour un volume estimé à 6 millions de $\mathrm{m}^{3}$.L'implantation de zones de stockage avec neuf bassins de réception des sédiments dragués, ont été réalisés (KAZI AOUAL - BENSLAFA et al., 2014 ; KAZI AOUAL - BENSLAFA et al., 2015).Cette solution a été préconisée par les deux projets de recherche auxquels nous avions participé : le projet national de préservation des ressources en 2011 ainsi que le projet de recherche intitulé "Conception et aménagement d'aires de stockage des sédiments en attente de valorisation" 2012-2014. Nous avions préconisé la solution intermédiaire du modèle $\mathrm{C} 2 \mathrm{C}$ qui est le modèle Cradle to Gate. Le modèle Cradle to Gate répondait exactement à notre première préoccupation : la préservation de l'environnement par le stockage des sédiments dans des zones spécialement aménagées (bassins) et proches du barrage pour une éventuelle exploitation et en attente de valorisation.

Cette réalisation prouve la prise de conscience des responsables gestionnaires et décideurs à réduire les impacts environnementaux négatifs. Les bassins ont été réalisés sur un terrain surélevé par rapport à la côte du plan d'eau du barrage avec une dénivelée de $10 \mathrm{~m}$ représentée sur le plan (figure 5). Cela permet d'évacuer l'eau après décantation des sédiments. Dans cette option de gestion des sédiments, les responsables ont opté pour la solution de la déshydratation naturelle des sédiments déposés et qui semble la plus économique dans l'immédiat. D'autres solutions peuvent être développées.

\section{Perspectives pour le développement d'une économie circulaire dans la gestion des sédiments de dragages}

Le développement d'une économie circulaire pour une gestion durable de la ressource en eau des barrages fait face à certains freins que les parties prenantes devront lever ; Les gestionnaires des barrages devront rechercher : 


\section{Thème 6 - Gestion durable des zones littorales et estuariennes}

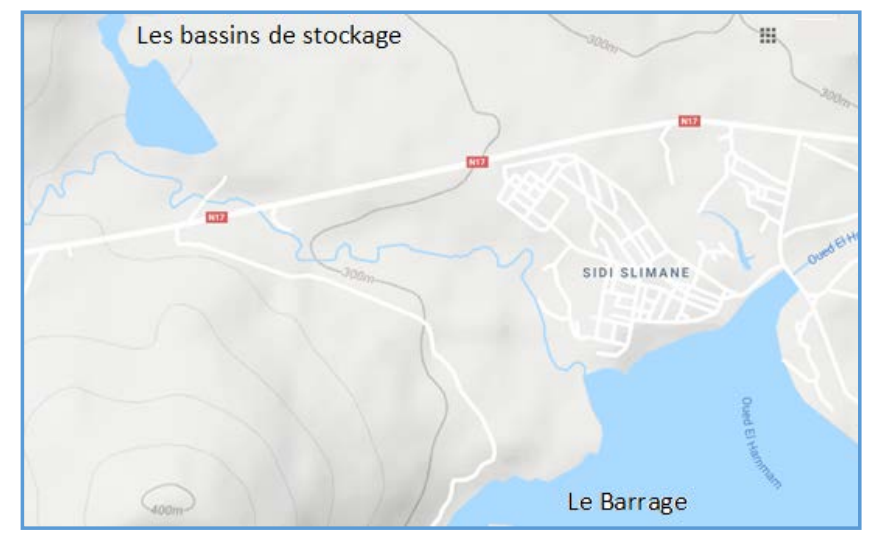

Figure 5.Situation topographique du barrage et des bassins de stockage, (Google Maps).

1. La mise en œuvre du modèle $\mathrm{C} 2 \mathrm{C}$ qui se rapproche le plus de l'économie circulaire. Pour cela des changements de conception du produit issu des sédiments de dragage sont nécessaires à l'état initial ou après traitement.

2. La recherche d'une valeur ajoutée du produit par des investissements dans les services de recherche et développement ainsi que dans des petites infrastructures pour l'exploitation des solutions viables du sédiment déposé dans les bassins de décantation.

3. Le rapprochement, au niveau de la zone d'influence, avec les acteurs économiques susceptibles d'utiliser le produit et répondre à la demande.

Ces trois volets de la gestion des sédiments de dragage favorisent une gestion optimale des retenues de barrage et la préservation de la ressource en eau. Chacun des trois volets représente des perspectives de recherche dont certaines ont donné des résultats intéressants en laboratoire mais qui restent à confirmer à l'échelle industrielle.

Les recherches en Algérie portant sur la valorisation des sédiments de dragage sont réalisées en laboratoire et peu appliquées à l'échelle industrielle. La deuxième prise de conscience des responsables afin d'arriver à une gestion durable et une économie circulaire est de permettre la confirmation au niveau industriel des résultats obtenus en laboratoire. Celle-ci prendra en compte toutes les parties prenantes en appliquant le modèle de l'ADEME représenté en figure 2:

1. Les gestionnaires des barrages pour la gestion de la ressource en eau et une intégration de la gestion des sédiments de dragage,

2. L'offre des acteurs économiques tels que les cimenteries de la région et le développement des petites industries de proximité pour la fabrication de produits céramiques,

3. Le comportement responsable des utilisateurs des ressources naturelles. Le barrage de Fergoug qui est envasé à 94\% représente un énorme gisement à exploiter, quant au 


\section{XVIèmes Journées Nationales Génie Côtier - Génie Civil \\ Le Havre 2020}

barrage de Bouhanifia envasé à $54 \%$, la solution préconisée est de maintenir le dragage en continu pour alimenter la fabrication de produits à partir des sédiments dragués.

\section{Conclusions}

Le travail d'investigation se poursuit pour l'élaboration de la fiche d'identité des SD pour intégrer l'économie circulaire à travers le modèle $\mathrm{C} 2 \mathrm{C}$. C'est ainsi qu'après avoir tracé un rapide aperçu sur l'EC et le modèle C2C, nous sommes concentrés sur les deux barrages celui de Fergoug et celui de Bouhanifia qui présentent un fort taux d'envasement et donc des gisements importants de sédiments à valoriser. Les résultats de nos recherches pour la protection de l'environnement ont eu pour application la réalisation des bassins de stockage des sédiments dragués en attente de valorisation. Enfin, nous avons apporté un rapide éclairage sur les freins au développement de l'EC en Algérie qui devront être levés par la valorisation des sédiments de dragage à l'échelle industrielle pour la confirmation des résultats obtenus en laboratoire afin d'aboutir à une gestion optimale des barrages et la préservation des ressources naturelles.

\section{Références bibliographiques}

ADEME -Agence de l'environnement et de la maîtrise de l'énergie- (2014). Fiche technique : l'économie circulaire, notions, $10 \mathrm{p}$.

ANBT (2015). Agence Nationale des Barrages et Transferts http://www.mre.gov.dz/presentation-de-lanbt/ (Consulté le 15 janvier 2020).

BRENNAN G., TENNANT M., BLOMSMA F. (2015). Business and production solutions: Closing the loop, in Kopnina, H. and Shoreman-Ouimet, E. (Eds). Sustainability: Key Issues. Earth Scan, pp 218-245.

COP21 (2015). Conférence de Paris de 2015 sur les changements climatiques, Le Bourget,France, $21^{\mathrm{ème}}$ conférence des 196 parties réunies.

EMF-ELLEN MACARTHUR FOUNDATION (2013). Towards the circular economy, Part 1: Economic and business rational for an accelerated transition, Ellen MacArthur Foundation, Isle of Wight, UK, 98 p.

KAZI AOUAL-BENSLAFA F., TOUHAMI K. (2019). The waste valorization and the circular economy in Algeria: an overview, $2^{\text {nd }}$ Euro-Mediterranean Conference for Environmental Integration, Sousse, Tunisia (in press 2019, 6 p).

KAZI AOUAL - BENSLAFA F., AMEUR M., MEKERTA B., SEMCHA A. (2014). Caractérisation des sédiments de dragage du barrage de Bouhanifia pour une

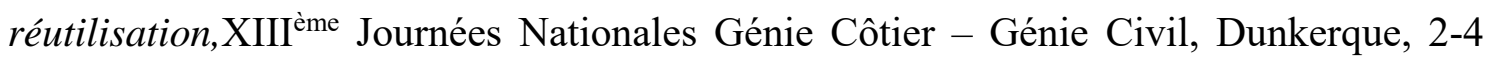
juillet 2014, France, pp 99-1006. https://doi.org/10.5150/jngcgc.2014.110

KAZI AOUAL - BENSLAFA F., AMEUR M., MEKERTA B., SEMCHA A. (2015). $L$ 'envasement du barrage de Bouhanifia : dragage et valorisation des sédiments, $\mathrm{CM}^{2}$ Conference 2015, November 25-27, Ferrara, Italy. https://doi.org/10.5150/cmcm.2015.032 


\section{Thème 6 - Gestion durable des zones littorales et estuariennes}

KORHONEN J., HONKASALO A., SEPPÄLÄ J. (2018). Circular economy: the concept and its limitations. Ecological Economics, Vol. 143(C), pp 37-46. https://doi.org/10.1016/j.ecolecon.2017.06.041

LE BISSONNAIS Y., LE SOUDER C. (1995). Mesurer la stabilité structurale des sols pour évaluer leur sensibilité à la battance et à l'érosion. Etude et Gestion des sols, 2,1, pp 43-56.

LENZEN M., MORAN D., KANEMOTO K., FORAN B., LOBEFARO L., GESCHKE A. (2012). International trade drives biodiversity threats in developing nations, Nature, Vol. 486, pp 109-112. https://doi.org/10.1038/nature11145

Mc DONOUGH W., BRAUNGART M. (2003). Towards a sustaining architecture for the 21 ${ }^{\text {st }}$ century: the promise of Cradle-to-Cradle design, Sustainable building and construction, UNEP Industry and Environment, Ind. Environ., Vol. 26 (2-3), pp 13-16 MERLI R., PREZIOSI M., ACAMPORA A. (2018). How do scholars approach the circular economy? A systematic literature review. Journal of Cleaner Production, Vol. 178, pp 703-722. https://doi.org/10.1016/j.jclepro.2017.12.112

MURRAY A., SKENE K., HAYNES K. (2017). The circular economy: an interdisciplinary exploration of the concept and application in a global context. J. Bus. Ethics, 140 (3), pp 369-380. https://doi.org/10.1007/s10551-015-2693-2

NAZET-ALLOUCHE D. (2016). La promotion de l'économie circulaire: quelles normes ? CERIC - DICE UMR 7318, Aix-Marseille Université, https://halshs.archivesouvertes.fr/halshs-01316181

PAULIUK S. (2018). Critical appraisal of the circular economy standard BS 8001:2017 and a dashboard of quantitative system indicators for its implementation in organizations, Resources, Conservation and Recycling, Vol. 129, pp 81-92. https://doi.org/10.1016/j.resconrec.2017.10.019

REMINI A. (2019). L'Algérie : de la boue au fond des barrages, que faire ? Algeria: the mud at the bottom of the dams, what to do? Larhyss Journal, ${ }^{\circ} 40$, pp 213-247.

SEMCHA A. (2015). Valorisation de la vase de barrage dans la production d'une brique, Présentation orale, Séminaire sur les enjeux des sédiments et leur valorisation dans les matériaux de construction, Université de Cergy-Pontoise, France.

TOUAHIR S., ASRI A., REMINI B., SAAD H. (2018). Prédiction de l'érosion hydrique dans le bassin versant de l'oued Zeddine et de l'envasement du barrage Ouled Mellouk (Nord-Ouest algérien), Géomorphologie : relief, processus, environnement, Vol. 24(2), pp 167-182. https://doi.org/10.4000/geomorphologie.12083

WWF -World Wildlife Fund- (2014). Living Planet, Report 2014, Species and spaces, people and places, chapitre1: The state of the Planet. Rapport $180 \mathrm{p}$. 